\title{
Römisches Recht und lokale Rechtssysteme Der Schutz der hellenistischen parakatatheke in drei Textstellen der Digesten
}

In $2^{\text {nd }}$ century a.d. the Roman empire is by then a cosmopolitan reality, open to the Hellenistic uses' influences. The jurists come up against the difficults to interpret the many-sides and new provincial background with the conceptual and legal tools of Roman law: they adapt the usual means of protection to original case record, follow a real justice's aim and update the law. The achieved outcomes are proved by some Digest's passages, where cases of extraitalic world - that breaks in even the language, with the use of Greek - and not perfectly frameble in Roman typical agreement are put. D 16, 3, 26, 1 (Paul. 4 resp.), D 31, 34, 7 (Mod. 10 resp.) and D 32, 37, 5 (Scaev. 18 dig.) can be considered illustrative of described interpretational operation: they are three passages mit Hellenistic setting, united by clauses in Greek with a reference to Greek 'parakatatheke'.

Die kurzen Ausführungen, die hier vorgebracht werden sollen, konzentrieren sich auf drei Textstellen der Digesta Iustiniani, die als exemplarisch betrachtet werden, um die Modalitäten zu beschreiben, mit denen die Juristen verschiedener, aber angrenzender Epochen hinsichtlich der Rechtsprechung die Beilegung eines Konflikts zwischen zwei normativen Systemen erreichen dem römischen und dem lokalen Rechtssystem. ${ }^{1}$ Es soll kurz daran erinnert werden, dass die Frage im Rahmen der Umsetzung der constitutio Antoniniana bereits in der Rechtslehre diskutiert wurde und nach wie vor diskutiert wird. Die Diskussion ging dabei von den gegensätzlichen Positionen aus, die Mitteis und Schönbauer vertraten. Nach Mitteis Ansicht hat das römische Recht die einheimischen Bürgerrechte gewaltsam verdrängt, auch wenn diese offensichtlich in einigen partikularen Rechtsgewohnheiten überlebt haben. Schönbauer hingegen war der Meinung, das römische Recht habe die einhei-

\footnotetext{
1 TALAMANCA, Diritto e prassi 135ff.
}

mischen Provinzialrechte nicht verdrängt, sondern sei gemeinsam mit diesen zur Anwendung gekommen, so dass aus dem gleichzeitigen Fortbestehen der beiden Systeme eine Ausgestaltung der Rechtsgeschäfte entstanden sei, die aus beiden juristischen Traditionen geschöpft habe. ${ }^{2}$ Beide Positionen räumen die Möglichkeit eines Konflikts ein, da die Betroffenen bei der Wahl der juristischen Form für ihre Rechtsgeschäfte zu gleichen Teilen auf jede der beiden Rechtsordnungen zurückgreifen konnten. Im Fall der ersten Hypothese würde der Konflikt mutmaßlich vom Willen des Gewinners abhängen, lokale Schemata und Einrichtungen durch eigene zu ersetzen; im zweiten Fall hingegen würde der Konflikt nur zum Tragen kommen, wenn die Bandbreite der Verhandlungsfreiheit so groß wäre, dass sie gegen die Prinzipien verstoßen würde, die wir heute als „öffentliche Ordnung" bezeichnen und die vermutlich auch

\footnotetext{
${ }^{2}$ FrEZZA, Istituti ellenistici 87f.; MITTEIS, Reichsrecht 161; SCHÖNBAUER, Die Doppelbürgerschaft 343ff.; SCHÖNBAUER, Das römische Recht 369ff.
} 
schon im römischen Rechtssystem existent waren. ${ }^{3}$ Die aus dem Edikt von Caracalla resultierende Unterscheidung wird hier jedoch für die nachfolgenden kurzen Überlegungen nicht berücksichtigt. ${ }^{4}$ Vielmehr soll unter Beibehaltung einer diachronischen Perspektive in erster Linie die Haltung eines antonianischen Juristen untersucht werden, namentlich Cervidius Scaevola, im Anschluss daran die Positionen von zwei bedeutenden Vertretern der severianischen Rechtsprechung, Paulus und Modestin.

Das Vergleichsmoment der ausgewählten Textstellen - es handelt sich um D 32, 37, 5; D 16, 3, 26, 1 und D 31, 34, 7 - besteht im Bezug auf die parakatatheke, ein Rechtsgeschäft des griechischen und hellenistischen Rechts, das zahlreiche Formen annehmen und Fälle betreffen kann. 5

D 32, 37, 5 (Scaev. 18 dig.): "Codicillis ita scripsit:

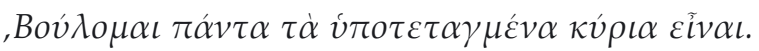

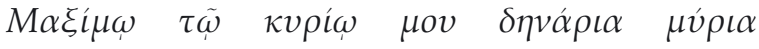

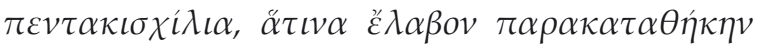

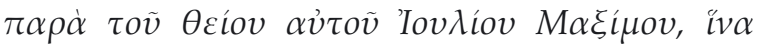

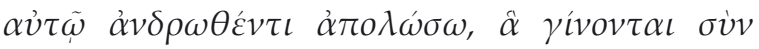

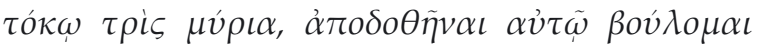

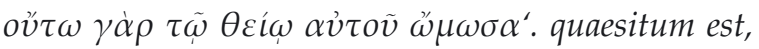
an ad depositam pecuniam petendam sufficiant verba codicillorum, cum hanc solam nec aliam ullam probationem habeat. respondi: ex his quae proponerentur, scilicet cum iusiurandum dedisse super hoc testator adfirmavit, credenda est scriptura."

Ein Erblasser fügt dem Testamentsnachtrag eine Bestimmung hinzu, nach der seinem Herren Maximus die fünfzehntausend Denaren zurückerstattet werden sollen, die er von dessen Onkel Julius Maximus bis zur Volljährigkeit des Begünstigten zur Verwahrung erhalten hatte. Es

\footnotetext{
3 TALAMANCA, Lineamenti 359ff.; 500ff.

${ }^{4}$ AmelotTI, Reichtsrecht 211ff.; TAlamancA, Lineamenti 600 .

${ }^{5}$ Wir finden das Wort (aber mit einer unfachlich Beteutung) parakatatheke auch in D 40, 5, 41, 4 (Scaev. 4 resp.).
}

handelt sich um eine Summe von dreißigtausend Denaren einschließlich der Zinsen. Der Erblasser fügt hinzu, dass dem Hinterleger Maximus auf diese Verpflichtung ein Eid geschworen wurde. Es stellt sich die Frage, ob die Worte des Testamentsnachtrags ausreichend sind, um die Summe vor Gericht einzufordern, da es außer diesen keinen anderen Beweis gibt. Scaevola erachtet es diesbezüglich als eindeutig, dass der testamentarischen Verfügung Glauben zu schenken sei, insbesondere angesichts der Tatsache, dass der Erblasser angegeben hat, darauf einen Eid geschworen zu haben. ${ }^{6}$

Ausgehend von dieser Fragestellung erschließt sich aus einer Reihe von Aspekten die Zugehörigkeit der fraglichen Rechtsverpflichtung zum lokalen Recht. Beispielhaft sei nur daran erinnert, dass die Klausel in griechischer Sprache verfasst wurde, sowie an die Art des Rechtsgeschäfts zwischen dem Erblasser und Julius Maximus als einer sogenannten irregulären Hinterlegung mit Zinsen gemäß einer in Griechenland gängigen Praxis. Weiterhin sprechen dafür die Ableistung eines Eids, der den rechtsgeschäftlichen Tatbestand mit einer für die griechische parakatatheke $e^{7}$ typischen treuhänderischen Konnotation belegt, außerdem die Funktion des Vertragsgeschäfts, die hier eher im Bereich der Vormundschaft als der Verwahrung zu verorten ist, gemäß einer Verwendung der parakatatheke, die laut einigen Autoren in bestimmten Fällen als simuliertes Rechtsgeschäft bezeichnet werden kann. ${ }^{8} \mathrm{Zu}$ guter Letzt sei auf das Vorhandensein vielfältiger Verweise auf die provinziale

\footnotetext{
${ }^{6}$ EISELE, Beiträge 24; BESELER, Beiträge 163; DELL'ORO, Costituzioni in greco 758; LITEWSKI, Studien 34, Anm. 9; LiTEWSKI, Dépôt irrégulier 243 und Anm. 107; KÜBLER, Griechische Tatbestände 188; FREZZA,

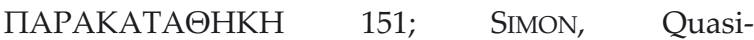

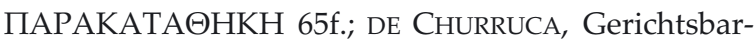
keit 322; VoCI, Diritto ereditario 324, Anm. 288; TALAMANCA, Clienti 550.

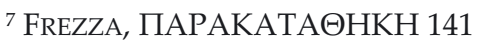

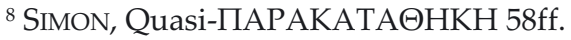


Welt in den Fallstudien von Scaevola verwiesen, was ein Unikum in den Zeugnissen der klassischen Rechtsprechung darstellt. ${ }^{9}$

Scaevola sieht sich gezwungen, eine vertraglich zwischen Lebenden vereinbarte Situation, die allerdings durch eben die eingegangene Verpflichtung $\mathrm{zu}$ einer Bestimmung mortis causa wurde, durch eine rechtliche Vorgehensweise zu schützen, die die Lehre teils als actio depositi, teils als petitio fideicommissi ausgelegt hat, und an deren Wirksamkeit und Gültigkeit aufgrund des Vorhandenseins von verba precativa sowie einer allgemeinen Klausel, die alle Bestimmungen des Dokuments schützt, kein Zweifel besteht. Mit anderen Worten würde die Frage angesichts des Fehlens von scripturae, die normalerweise die Hinterlegung eines depositum irregulare dokumentieren,10 auch die Möglichkeit eröffnen, die Testamentsnachträge als Beweismittel in einem iudicium depositi $\mathrm{zu}$ verwenden.

Es handelt sich also um eine Frage von überaus relevanter Beweiskraft, aus der sich jeweils weitere Fragen ergeben, nicht nur nach der Art des anzuwendenden Rechtsbehelfs, sondern auch nach den Legitimationsmustern - insbesondere der Aktivlegitimation - dieser Handlung, wenn man berücksichtigt, dass der Begünstigte der Erbschaft letztlich Maximus ist, der in formeller Hinsicht nichts mit dem Hinterlegungsvertrag zu tun hat.

Wenn man einmal von diesen Mustern absieht, ist der für unsere Zwecke wichtige Aspekt, dass Scaevola die Tatsache, dass sich eine inter vivos entstandene Verpflichtung den Prinzipien einer testamentarischen Verfügung annähert, als Frage der Rechtsprechung in toto aufnimmt und zu seinem Urteil gelangt, auch wenn es Elemente gibt, die nur schwerlich im klassisch römischen Recht zu verorten sind, wie die Entrichtung von Zinsen im Rahmen einer irregulären Hinterle-

\footnotetext{
9 TAlamanca, Particolarismo giuridico 74 .

${ }^{10}$ D 16, 3, 24, D 16, 3, 28 und D 16, 3, 26, 1-2.
}

gung, hinsichtlich deren Anerkennung der Jurist jedoch keinerlei Zweifel oder Verunsicherung zeigt.

Wenn man bedenkt, dass die Schaffensperiode des Cervidius Scaevola mitten im 2. Jahrhundert liegt, muss man die Flexibilität anerkennen, mit der er die Schutzinstrumente des römischen Rechts auf einen eindeutig lokal gefärbten Sachverhalt anwendet.

Ganz anders, wenngleich ebenso auf den Schutz von außerstädtischen Situationen ausgerichtet, präsentiert sich der Ansatz des Juristen Paulus im responsum zu D 16, 3, 26, 1.

D 16, 3, 26, 1 (Paul. 4 resp.): "Lucius Titius ita

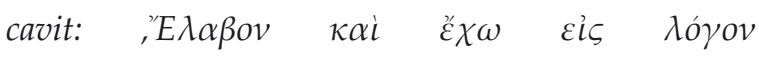
$\tau \alpha \rho \alpha \kappa \alpha \tau \alpha \theta \dot{\eta} \kappa \eta \varsigma \quad \tau \dot{\alpha} \quad \pi \rho о \gamma \varepsilon \gamma \rho \alpha \mu \mu \dot{\varepsilon} v \alpha \quad \tau o \tilde{v}$

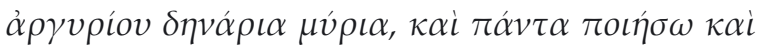

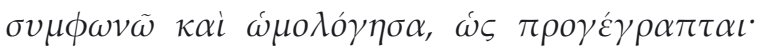

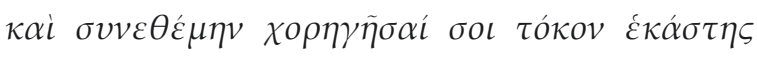

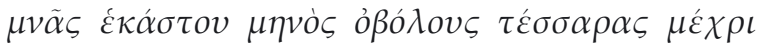

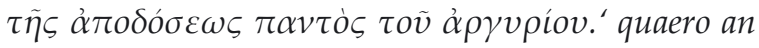
usurae peti possunt. Paulus respondit eum contractum de quo quaeritur depositae pecuniae modum excedere, et ideo secundum conventionem usurae quoque actione depositi peti possunt."

Ein gewisser Lucio Tizio hat sich verpflichtet, zehntausend Silberdenare anzunehmen und zu verwahren. Er verspricht, alle diesbezüglichen Absprachen einzuhalten, die in einem Schriftstück festgehalten wurden. Insbesondere sollte er „die Zinsen in Höhe von vier As-Münzen pro Monat Geldstück für Geldstück zurückzahlen, bis die gesamte Summe zurückerstattet wäre."11 An den Juristen wird die Frage gerichtet, ob man Zinsen verlangen könne. Paulus antwortet, dass dieser Vertrag über eine einfache Geldverwahrung hinausgeht und dass demnach gemäß des Übereinkommens mit dem Akt der Verwahrung auch Zinsen verlangt werden können. ${ }^{12}$

\footnotetext{
${ }^{11}$ SCOTTI, Deposito $80 \mathrm{ff}$.

12 GUARINO, Giusromanistica elementare 109 ; BONIFACIO, Ricerche sul deposito irregolare 90, 123, 134ff.;
} 
Der Passus wurde vielfach daraufhin analysiert, ob diese Figur, die in der Moderne gemeinhin auf das depositum irregulare zurückgeführt wurde, bei den klassischen römischen Juristen darstellbar war. Dieser Gedanke, der bereits auf den Anfang des 20. Jahrhunderts zurückgeht, ${ }^{13}$ wurde von diversen Autoren angefochten, ${ }^{14}$ für die das depositum irregulare ein Produkt der justinianischen Zeit war.

Die allgemeinen Begriffe dieser Kontroverse der Rechtslehre können hier nur angerissen werden, wir beschränken uns auf die Beobachtung, dass es keine formellen Hinweise darauf zu geben scheint, dass der Passus von Paulus abgeändert worden ist: daher muss davon ausgegangen werden, dass der severische Jurist es als legitim erachtete, den Tatbestand de qua mit dem Schutz einer mittels actio depositi ex fide bona getätigten Hinterlegung gleichzustellen. Weiterhin ist anzumerken, dass dieser Tatbestand eine provinziale Konnotation hat und daher den Gebrauch der griechischen Sprache und die beständigen Verweise auf die scripturae, bzw. auf die Schriftstücke bedingt, die - so darf man glauben nicht nur die Hinterlegung an sich bescheinigten, sondern auch die Zusatzvereinbarung der Entrichtung von Zinsen.

Nichtsdestotrotz wird ein tiefgreifender Unterschied zwischen der Haltung des severischen Juristen und der von Scaevola in D 32, 35, 7 geäußerten deutlich. Es wurde hervorgestellt, dass im Auszug von Scaevola keinerlei Zweifel an der Möglichkeit herrscht, die Zinsen zu fordern: in D 16, 3, 26, 1 hingegen besteht diesbezüglich Unsicherheit, da sich auf dieses Muster nicht nur das quaesitum, "quaero an usurae peti pos-

SARGENTI, Svolgimento 65f.; ADAMS, Haben die Römer 364; KlAMI, Mutua magis videtur 90ff.; LITEWSKI, Dépôt irrégulier 250ff.; GORDON, Observations 363ff.; SCOTTI, Deposito $85 \mathrm{ff}$.

${ }^{13}$ SEGRÈ, Sul deposito irregolare $197 \mathrm{ff}$.

${ }^{14}$ RotOndI, Appunti 72; MASCHI, Categoria dei contratti reali 382ff.; LONGO, Appunti 121ff.; ARANGIORuIZ, Istituzioni 312ff. sunt", bezieht, sondern auch das responsum, "usurae quoque actione depositi peti possunt". Die Vorrede, von der die Entscheidung des Paulus abhängt, enthält die Behauptung, dass eum contractum de quo quaeritur depositae pecuniae modum excedere, die von einigen als Widerspruch $\mathrm{zu}$ der vorgeschlagenen Lösung gelesen wurde. ${ }^{15}$

Der Diskurs von Paulus erscheint jedoch sinnvoll, wenn man ihn so paraphrasiert: normalerweise gehört die Entrichtung von Zinsen nicht zum Hinterlegungsvertrag; im Fall de quo aber ist die Sache anders, da der Vertrag sich auf die Hinterlegung mit einer zusätzlichen Vereinbarung der Zahlung von usurae bezieht. Eben weil der Vertragsinhalt anderer Art ist, ermöglicht es das rechtliche Vorgehen - das sich, wie schon gesagt wurde, ex fide bona darstellt - Verfahrensziele zu erreichen, die sonst ausgeschlossen wären. 16

Die Deutung des Paulus erscheint sogar noch bedenkenloser als die von Scaevola, da sie indirekt die Behauptung aufstellt, dass sich ein Instrument des klassischen römischen Rechts, wie die Hinterlegung, auch hinsichtlich eines Tatbestands des lokalen Rechts, der per se systematische Einordnungsschwierigkeiten im Bereich der typischen Bürgerrechte nahelegen würde, als wirkungsvoll erweisen kann.

Ist der Konflikt zwischen römischem Recht und lokalen Rechten im antonianischen Zeitalter in den Worten des Juristen stillschweigend implizit, so wird er bei Paulus augenscheinlich, eventuell in Folge des Edikts von Caracalla, mit den Auswirkungen auf das Verhältnis zwischen den Rechtsordnungen, auf die eingangs in diesem Artikel hingewiesen wurde.

Ebenso beispielhaft erscheint die von Modestin in D 31, 34, 7 dargelegte Antwort.

\footnotetext{
${ }^{15}$ MASCHI, Categoria dei contratti reali 391.

16 FIORI, Fides 259.
} 
D 31, 34, 7 (Mod. 10 resp.): "Titia cum nuberet Gaio Seio, dedit in dotem praedia et quasdam alias res, postea decedens codicillis ita cavit: ,Гáıov इéıov $\tau \dot{\alpha} v \quad \ddot{\alpha} v \delta \rho \alpha \quad \mu o v \quad \pi \alpha \rho \alpha \kappa \alpha \tau \alpha \tau i \theta \varepsilon \mu \alpha i \quad \sigma o l, \quad \tilde{\omega}$

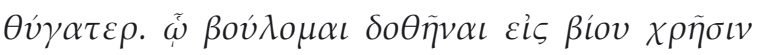

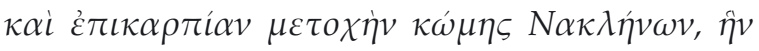

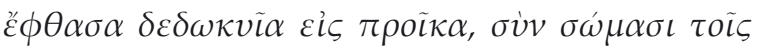
$\dot{\varepsilon} \mu \phi \varepsilon \rho о \mu \dot{\varepsilon} v o \iota \varsigma \tau \tilde{\eta} \pi \rho \circ \kappa i, \quad \kappa \alpha i \quad \kappa \alpha \tau \dot{\alpha} \mu \eta \dot{\delta} v$

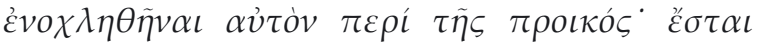

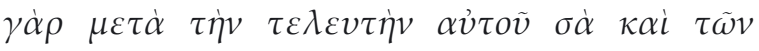

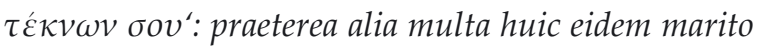
legavit, ut quamdiu viveret haberet. quaero, an propter haec, quae codicillis ei extra dotem relicta sunt, possit post mortem Gaii Seii ex causa fideicommissi petitio filiae et heredi Titiae competere et earum rerum nomine, quas in dotem Gaius Seius accepit. Modestinus respondit: licet non ea verba proponuntur, ex quibus filia testatricis fideicommissum a Gaio Seio, postquam praestiterit quae testamento legata sunt, petere possit, tamen nihil prohibet propter voluntatem testatricis post mortem Gaii Seii fideicommissum peti."

Man erzählt, dass Titia bei ihrer Hochzeit mit Gaius Seius als Mitgift einige Felder und andere Dinge in die Ehe einbrachte; vor ihrem Tod verfügte sie in einem Testamentsnachtrag, dass ihr Ehemann der Tochter anvertraut werden sollte: es wird das Verb $\pi \alpha \rho \alpha \kappa \alpha \tau \alpha \tau i \theta \varepsilon \mu \alpha \iota$ verwendet, also ,anvertrauen', vermutlich im weiteren Sinn als die ursprüngliche Bedeutung ,verwahren', erklärbar durch die sinnvolle Überlegung, dass nur eine Person zum Verwahrer bestimmt wird, der man vertraut. In der griechischen Klausel legt die Dame fest, dass der Teil eines gewissen vicus Nacolenorum, der dem Ehemann vorab als Mitgift eingebracht wurde, diesem als lebenslanges Nutzungsrecht gewährt werde, zusammen mit den auch als Mitgift eingebrachten Sklaven der Frau; und dass der Ehemann in keinster Weise an der Inanspruchnahme seiner ihm als Mitgift eingebrachten Güter gehindert werde. Diese Güter - so verfügte die Frau weiter - sollten nach dem Tod des Ehemanns in den Besitz der Tochter und deren Kinder übergehen.
Die Frau vermachte dem Ehemann darüber hinaus viele weitere Dinge, damit dieser sie behalte, solange er lebte. Es stellt sich die Frage, ob der Tochter und den Erben der Titia nach dem Tod von Gaius Seius eine petitio fideicommissi auch hinsichtlich der Dinge zusteht, die Gaius Seius zusammen mit der Mitgift erhielt. Modestin antwortet, dass auch wenn die Worte nicht zitiert sind, mit denen die Tochter der Erblasserin das Familienfideikommiss von Gaius Seius einfordern könnte, nachdem sie die Dinge in ihren Besitz gebracht habe, die ihr laut Testament vermacht wurden, dennoch kein Hindernis vorliegt, nicht gemäß dem Willen der Erblasserin nach dem Tod des Gaius Seius das Familienfideikommiss einzufordern. ${ }^{17}$

Wie Kübler eindeutig hervorgestellt hat, kann dieser Tatbestand nicht korrekt in die Schemata des klassischen römischen Rechts eingeordnet werden, da im Falle der Auflösung der Ehe durch den Tod der Frau die Mitgift, sofern adventicia, an den Ehemann übergeht, während im Falle einer Mitgift qua profecticia diese dem Vater zusteht oder jedenfalls dem Vorfahren, der sie als actio rei uxoriae eingebracht hat, wohingegen der Ehemann in diesem Fall Anrecht darauf hat, ein Fünftel des Vermögens für jedes gemeinsame Kind einzubehalten. ${ }^{18}$ Es scheint offensichtlich, dass im fraglichen Fall keine Indizien vorliegen, die auf eine dos profecticia hinweisen könnten: es wird nicht über Vorfahren oder sonstige Befugnisinhaber und auch nicht über eine Rückerstattung gesprochen, die Frau scheint direkte Rechtsinhaberin mit Verfügungsgewalt zu sein: man könnte hier also an eine dos adventicia von der Frau oder einem Dritten denken. Bei einer dos adventicia geht allerdings, wie schon erwähnt, die Mitgift beim Tod einer verheirateten Frau an den Ehemann über; ${ }^{19}$

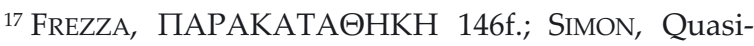

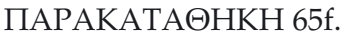

${ }^{18}$ KÜBLER, Griechische Tatbestände 188ff.

${ }^{19}$ BISCARDI, Diritto greco 101ff.; PAOLI, Famiglia 35ff.
} 
folglich wären die Bestimmungen von Titia allerdings sinnlos, nach denen sie mortis causa ein lebenslanges Nutzungsrecht für den Ehemann verfügt, der in diesem Fall bereits Besitzer des Gutes wäre und sich die Bestimmungen daher in ein legatum inutile auflösen würden. Auch wenn in diesem konkreten Fall zugegebenermaßen die Möglichkeit besteht, dass ein derartiges Rechtsgeschäft von Erblassern oder Bearbeitern ohne ausreichende Sachkenntnis ersonnen wurde, so erscheint es jedoch nicht plausibel, dass Modestin die praktische Sinnlosigkeit einer solchen Bestimmung nicht erkannt und dementsprechend gekennzeichnet haben sollte.

Der Fall passt übrigens perfekt in die Schemata des griechischen Rechts, wie in einigen Passagen des Gesetzes von Gortina (col. III, 17-24; col. III, 31-37; col. VI, 31-36) deutlich wird, und dessen Anwendung auch in nachfolgenden Epochen beibehalten wurde. ${ }^{20}$

Im fraglichen Fall kann man davon ausgehen, dass die Tochter der Erblasserin Besitzerin der Güter der Mutter ist, wenn ihr von derselben im wörtlichen Sinne, der Ehemann' anvertraut wurde, um ihr den letzten Wunsch zu gewähren, dem Ehemann lebenslanges Nutzungsrecht des aus der Mitgift bestehenden Vermögens einzuräumen und auch der - so liest man im lateinischen Satz, der kurz den Inhalt der Bestimmungen des Testamentsnachtrags wiedergibt - alia multa, also der nicht zur Mitgift gehörigen Güter, über die er bis zu seinem Lebensende verfügen kann.

Eine weitere unklärbare Mehrdeutigkeit bei der wörtlichen Auslegung des Paragraphen, so wie er von den Kompilatoren überliefert wurde, besteht im Verhältnis zwischen dem Ehemann und der Tochter der Frau. Die terminologische Wahl der Frau, die die Tochter als $\theta \dot{v} \gamma \alpha \tau \varepsilon \rho$ bezeichnet, den Nutznießer hingegen mit seinem

\footnotetext{
${ }^{20}$ Auch MitTEIS, Reichsrecht 231ff.; TAUBENSCHLAG, Law 95ff.; MitTEIs, Grundzüge 219ff.
}

eigenen Namen anredet, Gaius Seius, und ihn dann als ihren Ehemann qualifiziert - lässt die Schlussfolgerung zu, dass Gaius Seius nicht der Vater von Titias Tochter ist: Gaius Seius wird durch den Testamentsnachtrag eine Erbschaft hinterlassen, als handele es sich bei ihm um einen beliebigen Dritten, obgleich sein Status als Ehemann ausschlaggebend dafür ist, dass die Güter konkret an der gleichen Stelle bleiben wie vor dem Tod der Frau. Man kann vielleicht hinzufügen, dass so für den Ehemann eine Form von ius retentionis zur Anwendung kommt.

An dieser Stelle soll nicht weiter auf die etwaige Bedeutung des quaesitum und des entsprechenden responsum eingegangen werden, es sei nur daran erinnert, dass die Überflüssigkeit des Antwortteils zwischen licet und petere possit in der Lehrmeinung hervorgehoben und dessen Legitimation als petitio fideicommissi sogar $\mathrm{zu}$ Lebzeiten des Gaius Seius abgesprochen wurde, um dieselbe jedoch im anschließenden Teil zwischen tamen und Textende für die Zeit nach seinem Tod zu bestätigen. ${ }^{21}$ So kann man dem bereits von Kübler getätigten Hinweis folgen und annehmen, dass die Sorge der Tochter Titias um die zeitnahe Legitimation der Geltendmachung eines Familienfideikommiss eher darauf abzielte, Gaius Seius dazu zu zwingen, eine cautio fideicommissi servandi causa zu tätigen. ${ }^{22}$

Das von Titia oder von anderer Stelle in ihrem Auftrag formulierte quaesitum kann also folgendermaßen paraphrasiert werden: wenn das Nutzungsrecht ausgelaufen ist, das dem Ehemann von der Mutter für ihre Güter gewährt wurde, stellt dann die petitio fideicommissi das geeignete Rechtsinstrument dar, um nicht nur die zur Mitgift gehörigen Güter einzufordern, für die im Testamentsnachtrag ausdrücklich die Rückgabeverpflichtung geschrieben steht, sondern auch die nicht zur Mitgift gehörigen Güter,

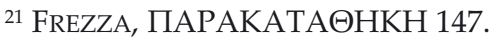

${ }^{22}$ KÜBLER, Griechische Tatbestände 189f.
} 
die der Tochter in ihrer Funktion als Erbin zustehen und über die sie laut testamentarischem Nachlass bis zu ihrem Tode verfügen kann?

Modestins Antwort auf diese Frage erscheint umsichtig und ausgewogen: der Jurist stellt sofort klar, dass bei den verba eine Leerstelle vorliegt, aufgrund derer der Anspruch nicht unmissverständlich bestimmt werden kann, schließt aber in dem Sinne, dass nihil [...] peti. Man kann annehmen, dass der Ausdruck nihil prohibet auf das Fehlen von normativen Hindernissen anspielt, oder besser gesagt auf das Fehlen von Auslegungsunterschieden zwischen den verschiedenen Rechtssystemen; es besteht also eine Kompatibilität zwischen dem Rechtsbehelf der petitio fideicommissi und der Lösung, die zur Anwendung kommen soll, falls der Tatbestand vom Juristen mit Verweis auf die voluntas testantis legitimiert wird.

Der Jurist Modestin, bekannt für seine provinziale Herkunft, die auch durch die Abfassung eines ganzen Werkes, der libri de excusationibus, auf Griechisch deutlich wird ${ }^{23}$ - befindet sich in D 31, 34, 7 in der Position, über einen Fall entscheiden zu müssen, der offensichtlich lokalem Recht unterliegt. Ihm wird die Frage gestellt, ob sich Bestimmungen, für die diametral gegensätzliche Prinzipien als die in Rom üblichen gelten, auf einen ex fideicommisso zu schützenden Sachverhalt zurückführen lassen. In der Tat ist das besprochene Verfahrensmittel, die Familienkommiss, eins der flexibelsten und anpassungsfähigsten überhaupt, sogar bezüglich der komplexesten Rechtsgeschäfte, in denen die fides als bestimmendes Merkmal fungiert. Es handelt sich um denselben Behelf, den auch Scaevola in D 32, 37, 5 vorschlägt, wenngleich mit einem teilweise divergierenden Deutungsansatz der Testamentsklauseln: soeben wurde deutlich gemacht, dass Paulus sich der Anomalie des besprochenen Rechtsgeschäfts im Vergleich zum römischen Recht bewusst war, wohingegen sich sein Meister Modestin eher nachgiebig zeigt, so dass der Konflikt in seiner Antwort nur aus der umsichtigen aber klaren Feststellung nihil prohibet hervorgeht. Darin kann man eventuell den Verweis auf eine Kategorie von obligatorischen Vorschriften des römischen Rechts lesen, deren Einordnung schwierig bleibt, aber in der man vielleicht ein Prinzip ausmachen kann, nachdem die voluntas testantis respektiert wird.

Abschließend lässt sich feststellen, dass die analysierten Textstellen auf einen Konflikt zwischen den verschiedenen Rechtsordnungen hinweisen, der zu antonianischer Zeit noch nicht offenkundig war, in den Entscheidungen der Rechtsprechung im Zeitalter Severians jedoch immer deutlicher hervortrat: in diesem Konflikt zwischen verschiedenen objektiven Rechtsordnungen fällt die schlichtende Rolle den Juristen zu. Diese ermöglichen durch den Schutz von Situationen, die durch andere als die römischen Rechtsordnung geregelt werden, eine wechselseitige Osmose der Systeme, so dass letztlich ein neues Rechtssystem entsteht, das sich nicht so sehr als Verbund, sondern vielmehr als eine Neuerung zur Lösung von Interessenskonflikten darstellt, und in Folge in der Rechtsprechung zur praktischen Anwendung kommt.

\section{Korrespondenz:}

\author{
Alessia Spina \\ Università degli Studi di Milano-Bicocca \\ Piazza dell'Ateneo Nuovo 1, 20151 Milano, Italia \\ alessia.spina1@unimib.it
}




\section{Abkürzungen:}

BIDR Bollettino dell'istituto di diritto romano

col. Kolumne

RIDA Revue Internationale des droits de l'antiquité

SDHI Studia et documenta historiae et iuris

\section{Literatur:}

Bertrand ADAMS, Haben die Römer Depositum irregulare und Darlehen unterschieden? in: SDHI 28 (1962) 360-371.

Mario AMELOTTI, Reichtsrecht, Volksrecht, Provinzialrecht. Vecchi problemi e nuovi documenti, in: SDHI 65 (1999), 211-215.

Vincenzo ARANGIO-RUIZ, Istituzioni di diritto romano (Napoli ${ }^{14} 1987$ ).

Gerhard BESELER, Beiträge zur Kritik der römischen Rechtsquellen, Bd. 3 (Tübingen 1913).

Arnaldo BISCARDI, Diritto greco antico (Milano 1982).

Franco BONIFACIO, Ricerche sul deposito irregolare, in: BIDR 49/50 (1947) 80-152.

Juan DE CHURRUCA, Die Gerichtsbarkeit des praefectus urbi über di argentarii im klassischen römischen Recht, in: ZRG RA 108 (1991) 304-324.

Aldo DELL'ORO, Le costituzioni in greco nei frammenti dei Digesta, in: Studi in onore di GaetanoScherillo, Bd. 2 (Milano 1972) 749-760.

Fridolin EISELE, Beiträge zur Erkenntniss der Digesteninterpolation. Zweiter Beitrag, in: ZRG RA 11 (1890) 1-29.

Roberto FIORI, Fides e bona fides. Gerarchia sociale e categorie giuridiche, in: Roberto FIORI $(\mathrm{Hg}), \mathrm{Mo}-$ delli teorici e metodologici nella storia del diritto privato (Napoli 2008) 237-260.

Paolo FrezZA, Istituti ellenistici nei testi del Corpus iuris civilis, in: Studi in onore di Vincenzo Arangio-Ruiz, Bd. 4 (Napoli 1953) 209-224 = Scritti di Frezza, 2 (Romae 2000) 87-102.

Paolo FrEZZA, ПAPAKATA@HKH, in: Symbolae Raphaeli Taubenschlag dedicatae, Bd. 1 (Vratislaviae-Varsaviae 1956) 139-172.

William M. GORDON, Observations on depositum irregulare, in: Studi in onore di Arnaldo Biscardi, Bd. 3 (Milano 1982) 363-372.

Antonio GUARINO, Giusromanistica elementare (Napoli 2002).

Hannu Tapani KLAMI, „Mutua magis videtur quam deposita“. Über die Geldverwahrung im Denken der römischen Juristen (Helsinki, 1969).
Bernhard KÜBLER, Griechische Tatbestände in den Werken der kasuistischen Literatur, in: ZRG RA 28 (1907) 174-210.

W. LITEWSKI, Le dépôt irrégulier 1, in: RIDA 21 (1974), 215-262.

Wieslaw LITEWSKI, Studien zur Verwahrung im römischen Recht (Warszawa-Kraków 1978).

Carlo LONGO, Appunti sul deposito irregolare, in: BIDR 18 (1906) 121-156.

Tommaso MASIELLO, I libri de excusationibus di Erennio Modestino (Napoli 1983).

Carlo Alberto MASCHI, La categoria dei contratti reali: corso di diritto romano (Milano 1973).

Ludwig MitTeIs, Grundzüge und Chrestomathie der Papyruskunde, Bd. 2: Juristischer Teil, Erste Hälfte: Grundzüge (Leipzig-Berlin 1912).

Ludwig MitTEIS, Reichsrecht und Volksrecht in den Österlichen Provinzen des römischen Kaiserreichs (Leipzig 1935).

Ugo Enrico Paoli, Famiglia (diritto attico), in: Novissimo Digesto Italiano 7 (Torino 1961) 35-42.

G. RotONDI, Appunti sulla teoria romana del deposito, in: DERS., Scritti giuridici, Bd. 2 (Milano 1922) $1-136$.

Manlio SARGENTI, Svolgimento dell'idea di contratto nel pensiero giuridico romano, in: Iura 39 (1988) 24-74.

Ernst SCHÖNBAUER, Die Doppelbürgerschaft im römischen Reiche und ihre Wirkung auf die Rechtsentwicklung, in: Anzeiger der Phil.-hist. Kl. der ÖAW 17 (1949) 343-369.

Ernst SCHÖNBAUER, Das römische Recht nach 212 in ausschließlicher Geltung?, in Anzeiger der Phil.hist. Kl. der ÖAW 17 (1949) 369-389.

Francesca ScotTI, Il deposito nel diritto romano. Testi con traduzione italiana e commento (Torino 2008).

Gino SEGRÈ, Sul deposito irregolare in diritto romano, in: Bullettino dell'Istituto di Diritto Romano 19 (1907) 197-234.

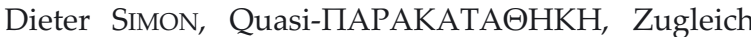
ein Beitrag zur Morphologie griechischhellenistischer Schuldrechtstatbestände, in: ZRG RA 82 (1965) 39-66.

Mario TAlamanca (Hg.), Lineamenti di storia del diritto romano (Milano ${ }^{2} 1989$ ).

Mario TALAMANCA, Diritto e prassi nel mondo antico, in: Isabella PIRO (Hg.), Règle et pratique du droit dans les réalités juridiques de l'Antiquité (Soveria Mannelli 1999).

Mario TAlamancA, Particolarismo giuridico ed unità della cultura giuridica nell'esperienza romana, in: Diritto generale e diritti particolari nell'esperienza 
storica. Atti del Congresso internazionale della Società italiana di Storia del diritto (Roma 2001) 9276.

Mario TAlamanca, I clienti di Q. Cervidio Scevola, in: BIDR 103-104 (2000-2001) 483-702.
Raphael TAUBENSCHLAG, The Law of Greco-Roman Egypt in the Light of the Papyri. 332 b.C.-640 a.D. (New York, 1944).

Pasquale VocI, Diritto ereditario romano, Bd. 2: Parte speciale (Milano ${ }^{2} 1963$ ). 